\title{
BUENAS Y MALAS NOTICIAS EN LA \\ LITERATURA GRIEGA ANTIGUA: DE HOMERO A LA ORATORIA
}

\author{
Raquel Fornieles \\ Universidad Autónoma de Madrid. España
}

Resumen: Tomando como punto de partida el estudio de la familia léxica derivada

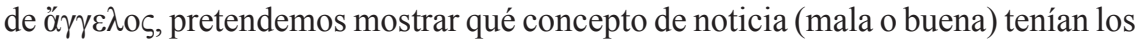
miembros de las sociedades representadas en los poemas homéricos, la tragedia, la comedia aristofánica, los tratados de los historiadores y la oratoria. El análisis del léxico evidencia que, pese a que la gran mayoría de las noticias son negativas, no existe un derivado de ó $\gamma \gamma \varepsilon \lambda$ ऽ que designe la mala noticia. Sin embargo, sí hay

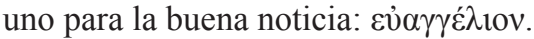

Palabras clave: ó $\gamma \gamma \varepsilon \lambda o \varsigma$ - buenas y malas noticias - léxico - literatura griega

\section{GOOD AND BAD NEWS IN ANCIENT GREEK LITERATURE: FROM HOMER TO ORATORY}

\begin{abstract}
This paper aims - taking as a starting point the lexical family derived from ö $\gamma \gamma \varepsilon \lambda o \varsigma$ - to show what concept of news (bad or good) had the members of the societies represented in Homer, tragedy, aristophanic comedy, the texts of historians and oratory. The analysis of the lexicon reflects that, although the vast majority of the news items are negative, there is no derivative of ö $\gamma \gamma \varepsilon \lambda \circ \varsigma$

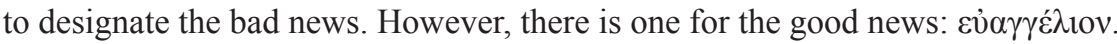

Keywords: $\alpha \gamma \gamma \varepsilon \lambda \mathrm{o}$ - Good news - bad news - lexicon - Greek Literature

Recibido: 19.07.2019 - Aceptado: 31.01.2020

Correspondencia: Raquel Fornieles

Email: raquel.fornieles@uam.es

Profesora Ayudante Doctora. Departamento de Filología Clásica.

Facultad de Filosofía y Letras

Universidad Autónoma de Madrid - Campus de Cantoblanco

ID Orcid: https://orcid.org/0000-0002-9252-4543 


\section{Introducción ${ }^{1}$}

7 odas las sociedades prestan atención continuamente a la noticia, elemento básico considerado incluso como un producto

1 de primera necesidad ${ }^{2}$. La antigua Grecia no era una excepción y, para entender qué era considerado noticia por los griegos, hemos realizado un estudio del léxico con el que ellos representaban su concepto de noticia ${ }^{3}$.

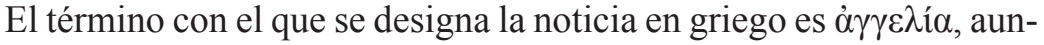
que debe tenerse en cuenta la reflexión de Lewis (1996, p.6), que sostiene que la verdadera noticia no es el hecho en sí mismo, sino lo que ocurre cuando dicho hecho se comunica y que, por tanto, la clave la daría el verbo $\alpha \gamma \gamma \varepsilon \dot{\varepsilon} \lambda \lambda \omega$, que es el término que se refiere al proceso. En nuestra opinión, tanto el análisis de $\alpha \gamma \gamma \varepsilon \lambda i^{4} \alpha^{4}$ como el de $\alpha \gamma \gamma \varepsilon \dot{\varepsilon} \lambda \lambda \omega$ resultan insuficientes por una sencilla razón: ambos derivan de ő́ $\gamma \gamma \varepsilon \lambda$ os, el mensajero, que no es otro que el responsable de transmitir la noticia. Así, la etimología nos ha conducido a iniciar el estudio con la palabra primitiva ǒ $\gamma \gamma \varepsilon \lambda \circ \varsigma \mathrm{y}$, a partir de ella, a hacerlo extensivo a todos sus derivados atestiguados en los autores que componen nuestro corpus.

En el examen del léxico hay algo que llama la atención: a pesar de que la mayoría de las noticias que se transmiten en la literatura griega son negativas, no existe en los textos analizados un término que nombre el concepto de mala noticia. Sin embargo, sí hay un derivado de ő $\gamma \gamma \varepsilon \lambda o \varsigma$ para

1 Trabajo realizado en el marco del Proyecto de Investigación FFI2015-65541C3-1-P.

2 Véanse Stephens (1988) o Harrison (2006), frente a la afirmación defendida por Schudson $(1978$, p.4) de que se trata de una invención norteamericana del siglo XIX.

3 Este trabajo es el desarrollo de una mínima parte de otro mucho más extenso - una tesis doctoral (Fornieles Sánchez, 2015) - en el que se indaga cómo se gesta nuestro concepto actual de noticia para mostrar que es una construcción mental (Shoemaker, 2006, pp.105-111) que, como otras, hemos heredado de los griegos.

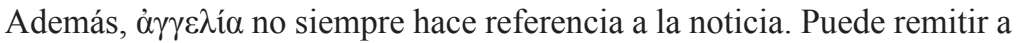
un mensaje o a una orden y solo podemos determinar de qué se trata atendiendo al contexto. 
la buena noticia: $\varepsilon \cup \jmath \alpha \gamma \gamma \varepsilon ́ \lambda ı v^{5}$. Nuestra hipótesis es que, a priori, la noticia siempre tiene connotaciones negativas en la literatura griega, el concepto de noticia que se detecta en el corpus estudiado es un concepto de noticia mala y, por tanto, no es preciso que reciba una denominación específica. Por el contrario, lo novedoso es que la nueva sea buena, de ahí que sí se haya creado un término concreto para designar las noticias favorables.

En el estudio individualizado de cada ejemplo no hemos examinado las concurrencias de forma aislada, sino los términos en su contexto verbal y, sobre todo, extraverbal (Coseriu, 1982, pp.314-317), que está muy relacionado con la pragmática y con toda una serie de valores y percepciones compartidos por los miembros de una sociedad. Por citar solo un caso, en la guerra una victoria es, en principio, una excelente noticia, pero la realidad es que solo lo es para los vencedores, nunca para los vencidos ${ }^{6}$.

Teniendo en cuenta lo expuesto, examinaremos en adelante qué concepto de noticia - mala o buena - tenían los miembros de las sociedades representadas en los poemas homéricos, la tragedia, la comedia aristofánica, los textos de Heródoto, Tucídides y Jenofonte y los discursos de Lisias, Isócrates, Demóstenes y Esquines, pues es en las obras de estos autores donde se localizan los derivados de ö $\gamma \gamma \varepsilon \lambda$ o que nos han permitido abordar esta investigación ${ }^{7}$.

\section{Homero}

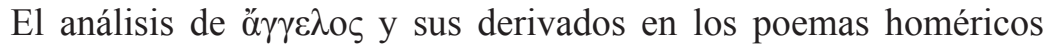

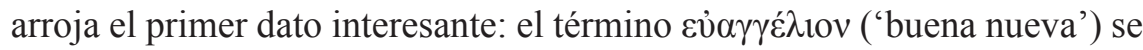
atestigua por primera vez en la literatura griega - dos veces - en la Odisea. Situémonos en el canto catorce. El porquero Eumeo, desolado por la incertidumbre, conversa con Odiseo, disfrazado de mendigo, y le asegura que

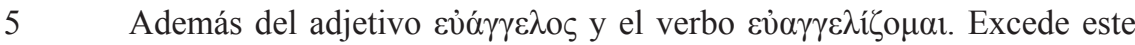

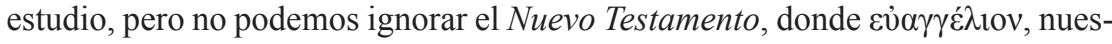
tro Evangelio adquiere un gran protagonismo. Remitimos, por ejemplo, a García Santos (2011).

6 Además, el transmisor de noticias ha de dar por supuesto que la nueva es relevante para su interlocutor, que afectará a su destinatario y tendrá consecuencias.

7 El corpus de la citada tesis lo forman Homero, la lírica coral, una selección de inscripciones, la tragedia, la comedia aristofánica, Heródoto, Tucídides y Jenofonte y los discursos de Lisias, Isócrates, Demóstenes y Esquines. 
ya no confía en que el regreso del héroe vaya a producirse, pues está convencido de que ha muerto. El propio Odiseo, aunque aún no está dispuesto a revelarle su identidad, trata de animarle:



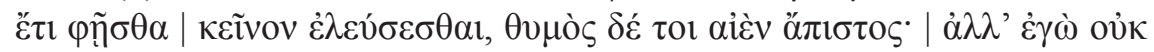

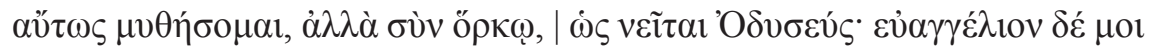

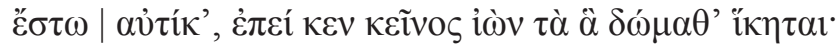

¡Amigo! Pues lo niegas por completo e incluso afirmas que aquel no regresará, en tu ánimo siempre desconfiado. Sin embargo, yo no referiré en vano, sino con un juramento, que volverá Odiseo. Y que por la buena nueva obtenga yo recompensa al punto, cuando él, tras llegar, haya entrado en su palacio ${ }^{8}$.

El porquero responde, escéptico (De Jong, 2001, p.351), con el mismo término:

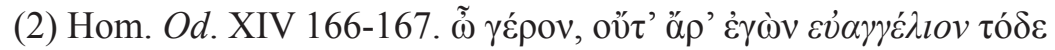

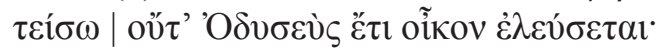

¡Anciano! Ni yo por la buena noticia te recompensaré, ni tampoco Odiseo a casa va a regresar.

Aunque Eumeo aún lo ignora, tiene ante sus ojos la buena nueva.

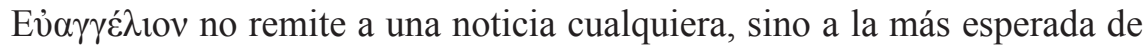
la Odisea y que tendrá importantes consecuencias, pues todos aguardan la llegada del héroe excepto los pretendientes, que morirán cuando todo se haya descubierto.

En la Ilíada la noticia más relevante no es la más deseada. En el can-


vv. 685-686): Héctor ha matado a Patroclo. Después, le pide que acuda

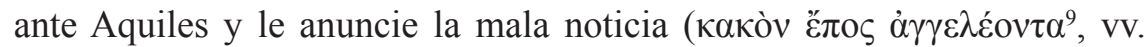

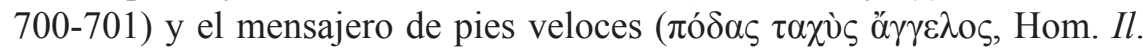

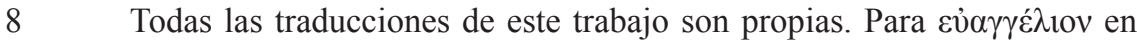
Homero seguimos los diccionarios. Véanse Ebeling (1885), LfgrE, LSJ o Bailly (1973). También, Merry, Ridell \& Monro (1886-1901), Merry (1907) o Bowie (2014).

9 Esta construcción solo aparece en este pasaje. En Hom. Il. XXIV 767 el


sino a un insulto. 
XVIII 2) informa al Pelida:



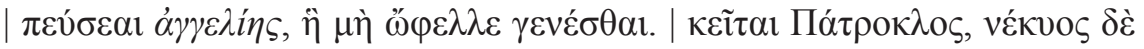

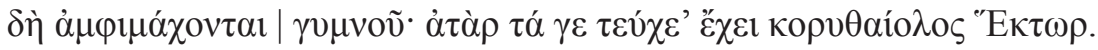

¡Ay de mí, hijo del valeroso Peleo! De una muy funesta noticia vas a enterarte, que ojalá no hubiera sucedido: yace Patroclo y están luchando alrededor de su cuerpo desnudo. Además, sus armas las tiene Héctor, de impetuoso penacho.

La importancia de la noticia es innegable, como evidencian las inmediatas consecuencias que de ella derivan: Aquiles depone su cólera y toma parte en el combate, lo que cambiará radicalmente el curso de la guerra.

A partir de pasajes como estos se observa que en los poemas homéricos no existe ningún término de la familia léxica que nos ocupa que aluda a la mala noticia. El matiz negativo se indica mediante los adjetivos que acompañan a la voz jonia $\alpha \gamma \gamma \varepsilon \lambda i ́ \eta$, como $\lambda v \gamma \rho \eta ́$ ('funesta') ${ }^{10}$ o $\dot{\alpha} \lambda \varepsilon \gamma \varepsilon v v \eta ́$

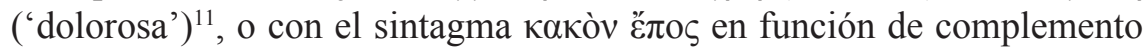

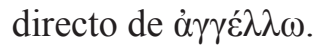

\section{La tragedia}

No podemos hablar de noticias en la tragedia griega sin referirnos al ö $\gamma \gamma \varepsilon \lambda o \varsigma$, figura creada para transmitirlas ${ }^{12}$. Aunque lo cierto es que la tragedia está repleta de noticias relacionadas con la muerte, la guerra o desgracias similares, solo hay tres derivados de ö $\gamma \gamma \varepsilon \lambda$ o $\varsigma$ que, a primera vista,

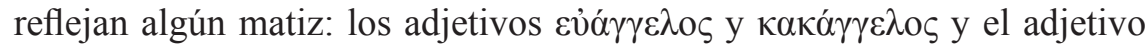

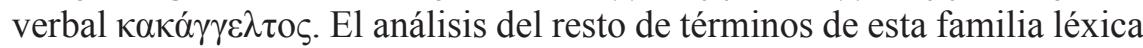
nos muestra que las noticias trágicas suelen ser desfavorables. Veamos los datos que ofrece el vocabulario.

10 Siempre en genitivo (cf. Hom. Il. XVII 642, Hom. Il. XVII 686 y Hom. Il. XVIII 19) o acusativo (cf. Hom. Il. XIX 337).

11 En dativo (cf. Hom. Il. II 787) o acusativo (Cf. Hom. Il. XVIII 17).

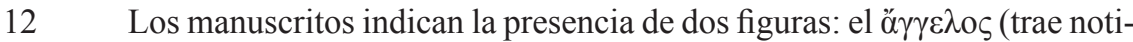

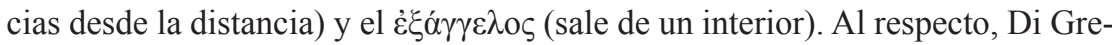
gorio (1967, pp.3-54). Un estudio detallado sobre el ŏ $\gamma \gamma \varepsilon \lambda o \varsigma$ trágico en Fornieles Sánchez (2018). 
Eủó $\gamma \gamma \varepsilon \lambda o \varsigma$ designa al mensajero de buenas nuevas. Esquilo lo atestigua cinco veces en Agamenón. En el prólogo, un vigía espera la llegada

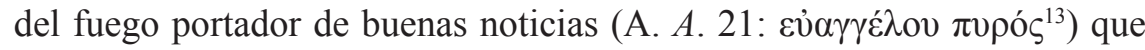
le indicará que Troya ha sido tomada. Después, Clitemnestra y el coro conversan y el coro asegura que, tanto si viene como mensajera de buenas

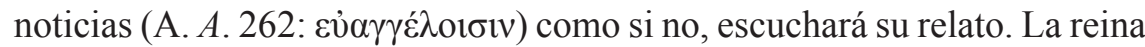
anuncia la nueva:

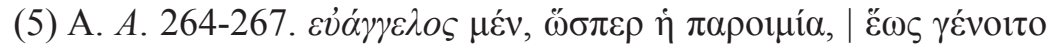

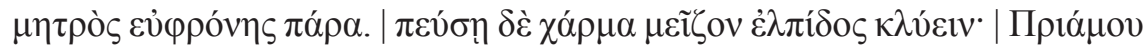

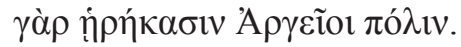

Como mensajera de buenas noticias, como dice el proverbio, que la aurora nazca de su madre, la noche. Te vas a enterar de una alegría mayor de oír que la esperanza: sí, han tomado los argivos la ciudad de Príamo.

En el siguiente ejemplo quien informa es un heraldo, un $\kappa \tilde{\rho} \rho \xi^{14}$. El coro, advierte que se aproxima coronado con ramos de olivo, señal de que porta un fausto mensaje (Perea Morales, 1993). Su primera noticia es favorable: el regreso de Agamenón. Sin embargo, aún debe comunicar la mala: Menelao ha desaparecido y nadie sabe si está muerto o sigue con vida. Así se lamenta:

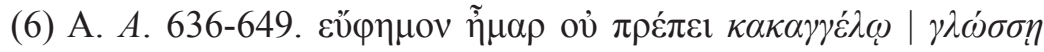



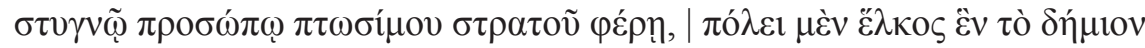

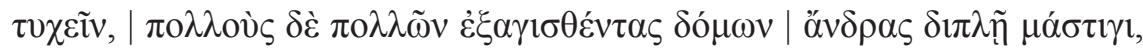



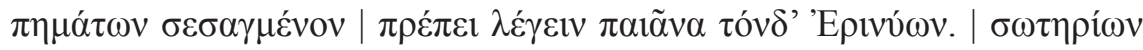

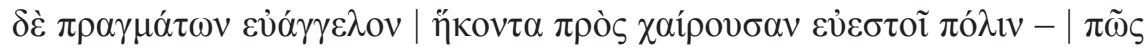



Un día favorable no conviene marcharlo con una lengua portadora de malas noticias. La honra a los dioses es diferente. Cuando un mensajero con el rostro entristecido lleva a una ciudad el odioso dolor de su ejército

13 El coro volverá a referirse así al fuego (cf. A. A. 475: $\pi$ vò̀ $\delta$ ' vं '



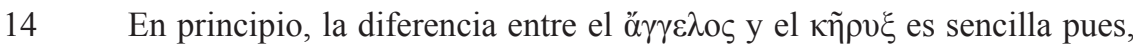

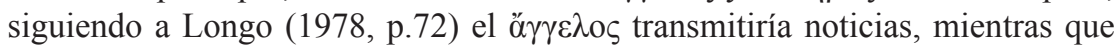
el $\kappa \tilde{\rho} \rho \xi$ se ocuparía, entre otras cosas, de comunicar mensajes y órdenes y de pregonar proclamas oficiales. 
aniquilado - para la ciudad una herida que a todos alcanza, muchos guerreros que han sido expulsados de sus casas por el doble látigo, grato a Ares, desgracia de dos lanzas, sangrienta yunta - en efecto, cargado de tales dolores, conviene que entone un peán a la Erinia. En cambio, al mensajero de buenas noticias de asuntos de salvación que ha llegado a una ciudad dichosa y feliz... ¿cómo voy a mezclar yo lo agradable con las desgracias contando la tempestad no falta de ira que sufrieron los aqueos?

El pasaje resulta realmente útil, pues permite establecer una dicotomía entre las nuevas consideradas negativas y las positivas. Desde el

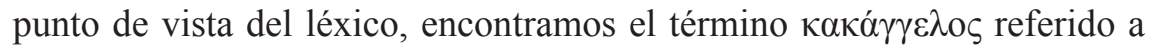
una lengua portadora de desgracias, que no es la suya propia porque, con lo que va a anunciar, manchará un día feliz. El heraldo también subraya

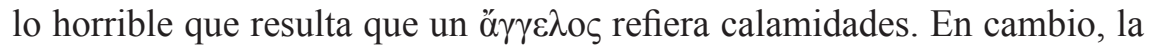
situación que ha experimentado poco antes era bien distinta, pues ha sido un mensajero de buenas nuevas (ยvoó $\gamma \gamma \varepsilon \lambda o v)$.

En estos versos se testimonia por primera y exclusiva vez en el corpus

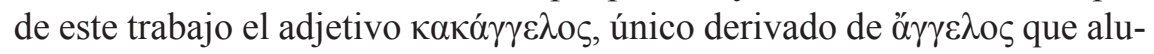

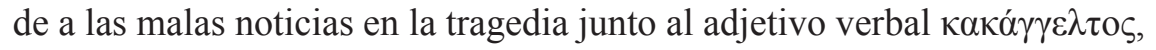
que también se documenta en una sola ocasión. El ejemplo se ubica en

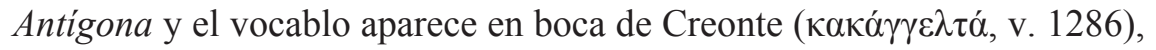
que se refiere al dolor causado por las malas noticias cuando se lamenta

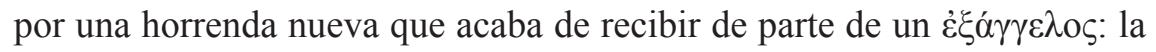
muerte de su esposa Eurídice.

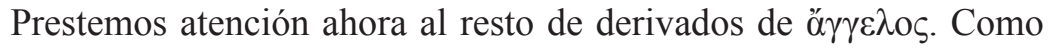

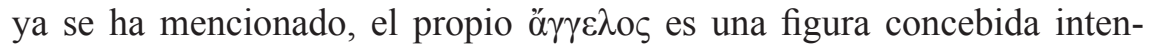
cionadamente para transmitir noticias. El ejemplo de (6) nos ha mostrado que, si las nuevas que va a anunciar son negativas, el ő $\gamma \gamma \varepsilon \lambda$ o se aproxima entristecido, y también lo hará con rostro sombrío ${ }^{15}$. Además, hay una con-

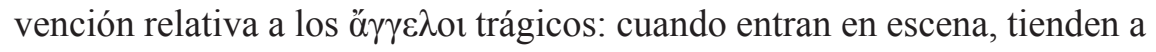
disculparse por las desgracias que vienen a comunicar (Mastronarde, 1979, p.69) como el ö $\gamma \gamma \varepsilon \lambda o \varsigma$ de $\operatorname{Persas}^{16}$.

$15 \quad$ Cf. E. $P h .1333$.

16 También el de Andrómaca, que se confiesa un infeliz por comunicar

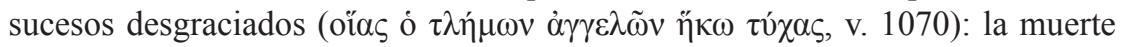

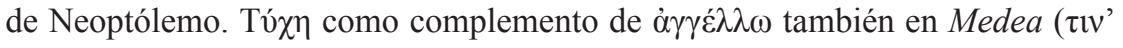

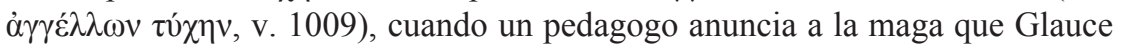






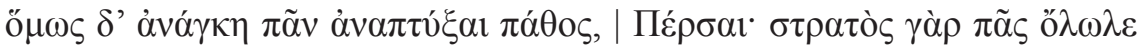
$\beta \alpha \rho \beta \alpha ́ \rho \omega v$.

¡Ay de mí! ¡Mi primera desgracia es anunciar desgracias! Y, sin embargo, es forzoso que os revele todo el desastre, persas. ¡Sí, el ejército bárbaro al completo ha perecido!

A veces son otros quienes los denominan 'mensajeros de desgracias'

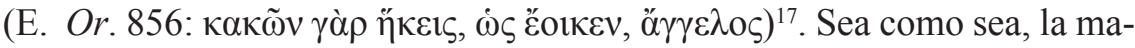
yoría de las noticias anunciadas por los ő $\gamma \gamma \varepsilon \lambda$ o o por el resto de personajes son negativas y se expresan de diversas maneras. Es frecuente encontrar

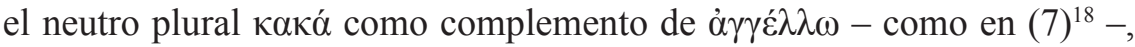
$\delta 1 \alpha \gamma \gamma \varepsilon \dot{\lambda} \lambda \omega^{19}$ o $\dot{\alpha} \pi \alpha \gamma \gamma \varepsilon \dot{\lambda} \lambda \lambda \omega^{20}$.

En otras ocasiones, el complemento directo es el singular какóv (E.

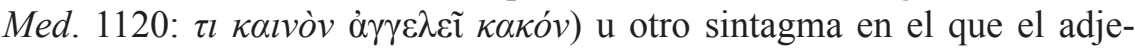
tivo какó $\varsigma$ expresa el tipo de nueva anunciada, como sucede cuando un ă $\gamma \gamma \varepsilon \lambda \mathrm{o}$ afirma en Ifigenia entre los tauros que acude con un cargamento

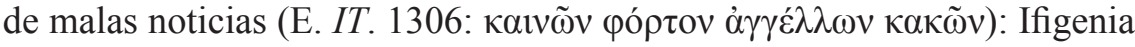
se ha fugado con los extranjeros. En un pasaje de la Electra de Eurípides,



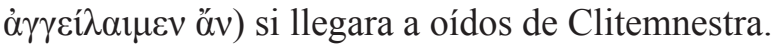

Prestemos atención de nuevo a la figura del mensajero. En conta-

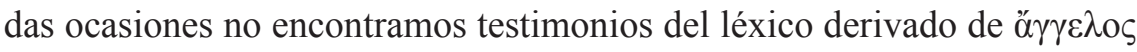
en sus intervenciones pero, en esos casos, se sirve de otras expresiones consideradas como una convención que se repite - junto a la presencia de ö́ $\gamma \varepsilon \lambda \mathrm{o} \varsigma$ y sus derivados - para dar paso al relato ${ }^{21}$. Una es el verbo $\eta ̋ \kappa \omega$ seguido de $\varphi \varepsilon ́ \rho \omega$ con acusativo o $\varphi \varepsilon ́ \rho \omega$ con acusativo dando lugar

ha aceptado sus regalos.

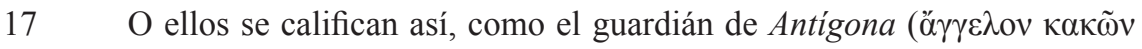
$\dot{\varepsilon} \pi \tilde{\omega} v$, v. 277).

18 Cf. también, E. Heracl. 54, E. El. 303 o E. HF. 1136.

19 Cf. E. Hel. 436.

20 Cf. A. Pers. 330.

21 Véanse Strohm (1959, pp.257-274), Mills (1981, pp.129-135), Hutchinson (1985, p.48) o Halleran (2005, pp.167-182). 


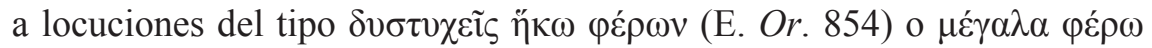

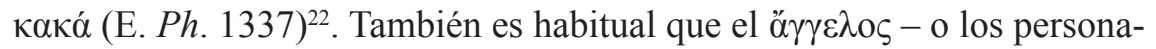
jes que mencionan sus noticias - se refiera a su función informativa con




de habla, como $\lambda \varepsilon ́ \gamma \omega, \varphi \eta \mu i ́, \varphi \rho a ́ \zeta \omega ~ o ~ \sigma \eta \mu \alpha i ́ v \omega^{23}$, como en (8), donde un auriga comunica nefastas nuevas, la muerte de los aliados y de Reso:

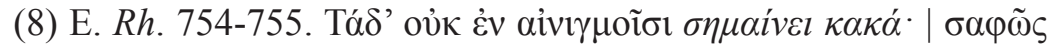

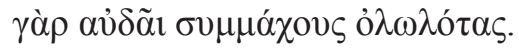

Estas palabras sin enigmas indican desgracias, pues claramente nos refiere que los aliados están muertos.

El examen del léxico nos muestra que solo en un pasaje de Orestes la noticia - Electra quiere salir de palacio sin ser vista y le pide al coro que le informe de cómo está la situación tras la puerta y le diga lo que quiere oír

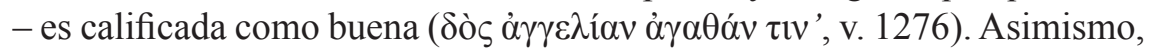

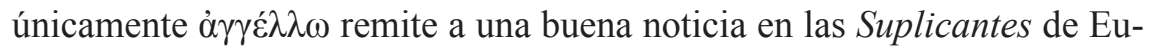
rípides, en un pasaje en el que va acompañado del adverbio eṽ y que hace referencia a la victoria de Teseo ( $\varepsilon \tilde{a} \dot{\alpha} \gamma \gamma \varepsilon \dot{\lambda} \lambda \lambda \varepsilon 1 \varsigma$, v. 641). Ninguno de los demás verbos de esta familia léxica presentan complementos que aludan a nuevas favorables y solo una de expresiones formulares que en ocasiones

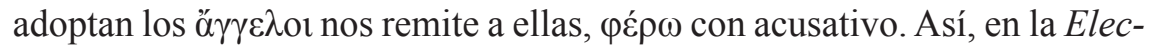
tra de Sófocles Orestes trama que un pedagogo anuncie una dulce noticia a

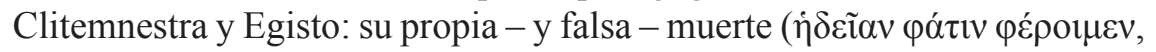

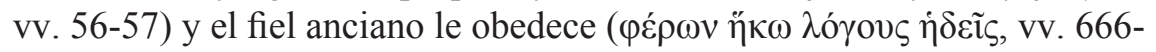



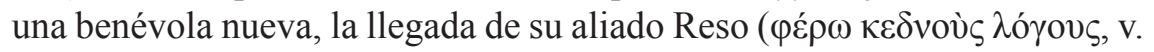
270) y, en Heraclidas, otro mensajero alegrará a Alcmena al comunicarle el triunfo de Yolao:

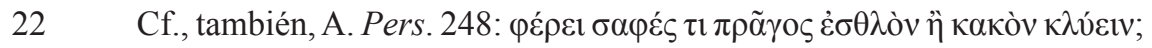

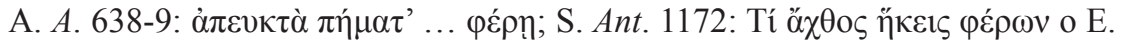

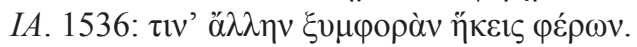

23 Seguimos a Mastronarde (2002, p.352) a partir de un verso de Medea

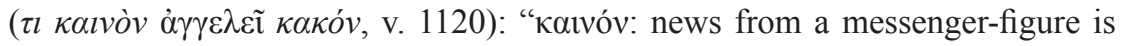

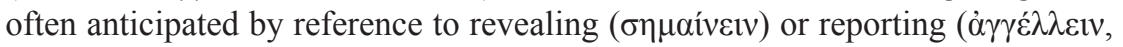

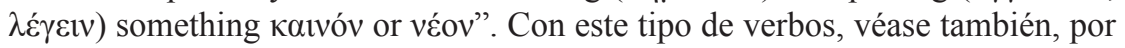

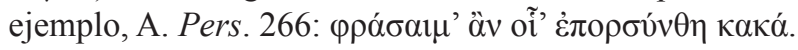




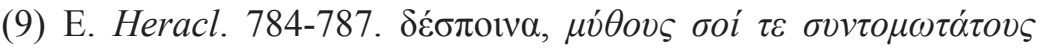

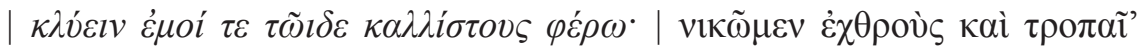

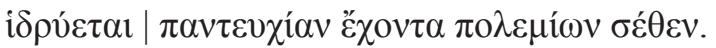

¡Señora! Traigo noticias para ti brevísimas de oír y, para mí, a tu lado, muy bellas: hemos vencido a los enemigos y se han erigido trofeos que contienen todas las armaduras de tus rivales.

\section{La comedia aristofánica}

Como es de suponer, no encontramos en la comedia aristofánica la solemnidad que caracteriza a la tragedia. Debemos tener en cuenta, además, el espíritu común que deriva de toda comedia política como la que nos ocupa, es decir, la crítica al poder establecido y a los dirigentes (Melero Bellido, 2000, pp.431-499). Pero no son los mandatarios las únicas víctimas del escarnio ${ }^{24}$. También son parodiados, además del propio Eurípides, los personajes y convenciones de la tragedia entre las que se encuentran el

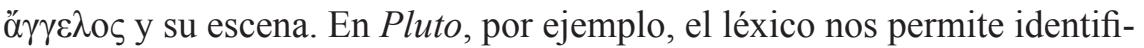
car a un mensajero. Los manuscritos no llaman ő $\gamma \gamma \varepsilon \lambda$ o a este personaje, pero como muchos de los ö $\gamma \gamma \varepsilon \lambda o 1$ del drama es un siervo ${ }^{25}$. Nos referimos a Carión, el sirviente de Crémilo, cuya irrupción es anunciada así por el corifeo:



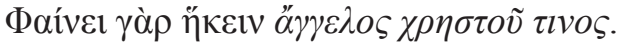

¿Qué sucede, queridísimo, con tus amigos? Pues parece que has venido como mensajero de algo provechoso.

La noticia que Carión entregará es, como muchas de las que transmiten los ő $\gamma \gamma \varepsilon \lambda$ o trágicos, todo un milagro: Pluto ha recobrado la vista. Lo que sigue es una escena de mensajero parodiada. Estamos ante una buena nueva a la que la esposa de Crémilo se referirá también con el acusativo del



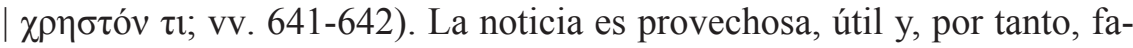
vorable. El examen del léxico nos muestra que, además de esta, hay otras

24 Según Brioso Sánchez (2006, pp.111-119), en la comedia la propensión paratrágica puede llegar a predominar por encima de la necesidad de la información.

25 En Avispas otro esclavo, Jantias, relata las consecuencias de un ridículo banquete en el que todos acabaron borrachos y del que él salió apaleado. 
buenas nuevas en la comedia.

En primer lugar, destaca en las obras de Aristófanes la presencia de

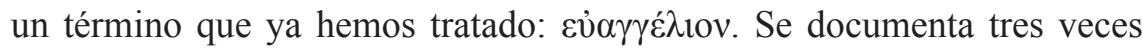
y siempre alude a noticias frívolas ${ }^{26}$. Los pasajes mostrados en (11) y

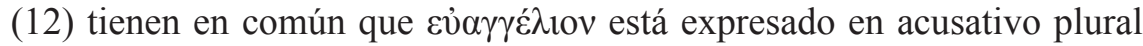
y expresa función semántica Causa de dos verbos que indican la acción de coronar al portador de las noticias en señal de agradecimiento. En (11) informa el Morcillero. Las nuevas que comunica las mostraremos más adelante - en el ejemplo (14) -, pues interviene otro de los términos objeto de nuestro estudio ( $\varepsilon \dot{\alpha} \alpha \gamma \gamma \varepsilon \lambda i \zeta o \mu \alpha \imath)$. Adelantaremos simplemente que su anuncio provocó, el entusiasmo de sus receptores:

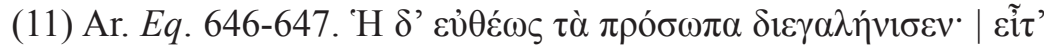

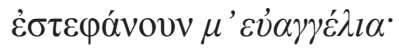

Inmediatamente los rostros se serenaron. Después, me coronaron por las buenas noticias.

El segundo pasaje nos lleva de nuevo a Pluto y al milagro del que Carión informa (Pluto ya no es ciego). La esposa de Crémilo muestra así su alegría:

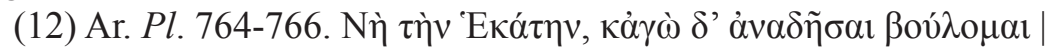

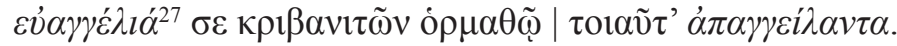

¡Por Hécate!, también yo quiero ceñirte por las buenas noticias que has anunciado con una cadena de panes cocidos al horno.

En ambos casos el transmisor de las nuevas es recompensado y

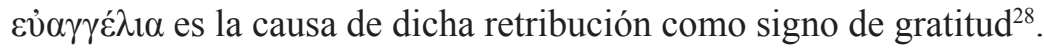

El siguiente ejemplo tiene un matiz especial. Volvamos a Caballeros. Tras las nuevas favorables que dieron lugar a su coronación, el Morcillero continúa relatando el debate que mantuvo con el Plafagonio y explica que su rival, para ganarse el favor del Consejo, hizo la siguiente proposición:

26 Dickson (2005, pp.213-214) sostiene que, dejando a un lado a Homero, $\varepsilon v \mathfrak{u} \gamma \gamma \varepsilon \lambda$ - se asocia a cuestiones de Estado, excepto en Aristófanes, que lo emplea para parodiar la solemnidad de los términos usuales en la transmisión de nuevas.

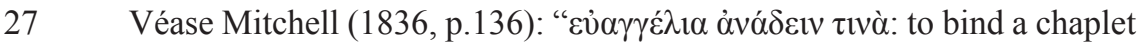
on a person's brow for the good news he brings".

28 Según Neil (1901, p.96) la corona es la recompensa habitual para el portador de nuevas favorables en Atenas. 


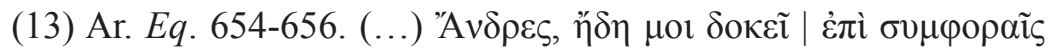

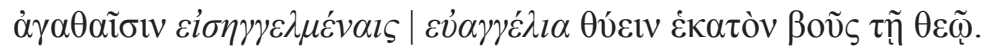

Señores, me parece bien en seguida, en honor de las coyunturas anunciadas, celebrar las buenas noticias con el sacrificio de cien bueyes a la diosa.

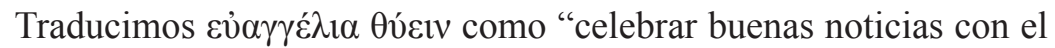
sacrificio" siguiendo la propuesta de $L S J$. El efecto cómico es evidente. El verbo $\theta v ́ \omega$, la acción de sacrificar víctimas en honor a la divinidad, impregna la escena de seriedad y respeto ${ }^{29}$, pero la parodia es indiscutible si tenemos en cuenta lo absurdo de la noticia que el Paflagonio pide que se agradezca, la siguiente:

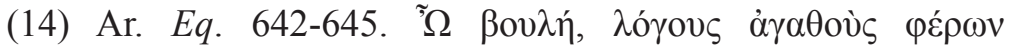

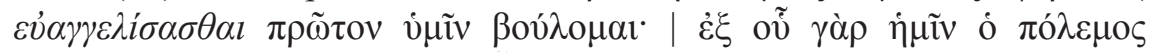

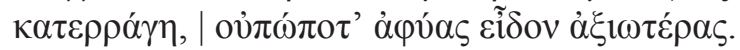

¡Oh, Consejo! Puesto que os traigo buenas noticias quiero anunciároslas en primer lugar a vosotros: en efecto, desde que la guerra nos estalló nunca vi las sardinas tan baratas.

El término con el que el Morcillero expresa que las nuevas son favorables es $\varepsilon \dot{u} \alpha \gamma \gamma \varepsilon \lambda i \zeta{ }_{0} \mu \alpha 1$, verbo que no se documenta Homero ni en la tragedia y que solo aparece en este pasaje en la comedia. Por otra parte, nos encontramos ante un caso de paratragedia ${ }^{30}$ llevada al extremo, pues el Morcillero ha llegado como ó $\gamma \gamma \varepsilon \lambda o \varsigma$ ante el coro y está representando una escena de mensajero cómica. Este pasaje está ubicado en pleno relato de mensajero y, ahora, está narrando su irrupción en el Consejo imitando

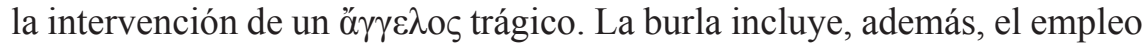
de un término de la familia léxica derivada de ó $\gamma \gamma \varepsilon \lambda o \varsigma$ y de una de las fórmulas usadas por los ó $\gamma \gamma \varepsilon \lambda$ or del drama: $\varphi \varepsilon ́ \rho \omega$ con un complemento en acusativo que, en este pasaje, resalta aún más el carácter favorable de las

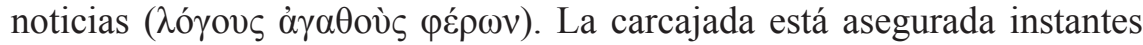
después cuando, en contraste con la seriedad de la que el Morcillero ha revestido la situación, anuncie la buena nueva: las sardinas están baratísimas.

29 Véase Sommerstein (1981, p.179): «Sacrified... in honour of these good tidings: as if news had come of a victorious battle or some similar occasion for national rejoicing".

30 Sobre la paratragedia remitimos principalmente a Rau (1967). 
Así, una vez más, el examen del léxico no permite saber si en la comedia las noticias son buenas o malas y lo cierto es que, aunque absurdas, predominan las positivas. En este sentido, además de los pasajes expuestos hasta el momento, debe destacarse la presencia en Aves de la expresión eṽ $\lambda \varepsilon \dot{\gamma} \varepsilon \varsigma^{31}$ (v. 1124) en boca de Pistetero como respuesta a la nueva que le ha

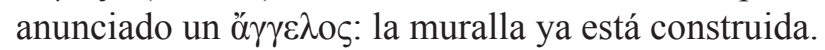

Respecto a las malas noticias, el análisis del vocabulario remite a dos pasajes. En (15), el corifeo de Acarnienses advierte de la llegada de un mensajero:



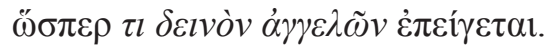

Por cierto, aquí se apresura uno alzando la ceja, como si fuera a anunciar algo terrible.

En este caso, el complemento de $\alpha \gamma \gamma \varepsilon \dot{\lambda} \lambda \lambda \omega$ es $\tau \imath \delta \varepsilon เ v o ́ v$, pero no alude a una noticia, sino a un mandato. En escena está Lámaco y a él se dirige el mensajero que, en esta ocasión, se comporta como un heraldo, pues le transmite un mensaje impresivo: "Los generales te ordenan partir hoy rápidamente con tus compañías de soldados y tus penachos" (vv. 10731074).

El otro pasaje es de Tesmoforias. Clístenes ya ha dado la noticia de que Eurípides iba a infiltrar a un pariente suyo entre las mujeres para enterarse de los planes que iba a llevar a cabo y, cuando el espía está a punto de ser descubierto, desconfía de las palabras de transmisor de las nuevas para intentar salir del paso. Sin embargo, el corifeo no duda de que Clístenes no

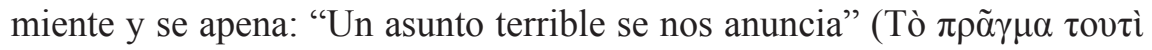

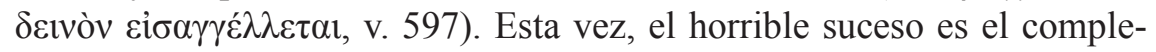
mento directo de $\varepsilon i \sigma \alpha \gamma \gamma \varepsilon \dot{\varepsilon} \lambda \lambda \omega$, otro de los verbos de la familia léxica que estamos tratando.

31 Traducimos $\varepsilon \tilde{u} \lambda \varepsilon_{\gamma} \gamma \varepsilon \varsigma$ como "buenas noticias" por analogía con otros

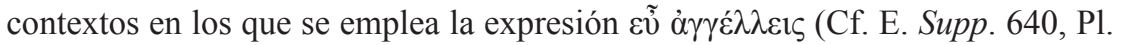
Tht. 144b o Pl. R. 432d). En Heródoto, con el verbo en aoristo (Hdt. VII 80: $\varepsilon \tilde{u}$

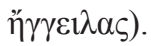




\section{Los historiadores}

Tampoco tenemos testimoniado en las obras de Heródoto, Tucídi-

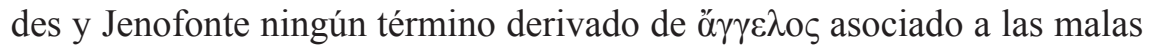

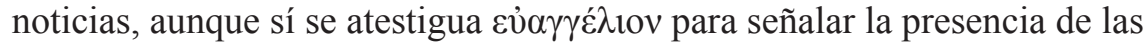
buenas. Solo Jenofonte lo emplea. Lo hace en dos ocasiones en Helénicas $\mathrm{y}$, en ambos casos, la recepción de las nuevas es celebrada con sacrificios ${ }^{32}$ - lo que confiere mayor gravedad a la situación - como muestra la presencia del verbo $\theta u ́ \omega^{33}$.

El primer pasaje nos sitúa en la batalla de las Arginusas. El espartano Eteonico, consciente de que el tiempo apremiaba y pronto tendría que enfrentarse a los enemigos, engañó a sus soldados:

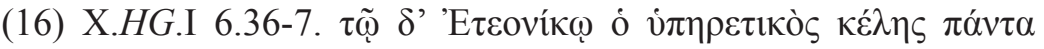

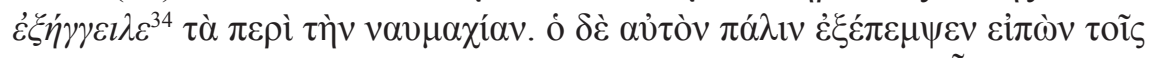

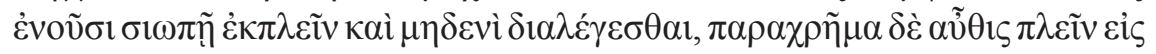



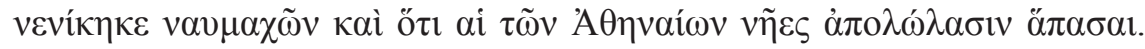

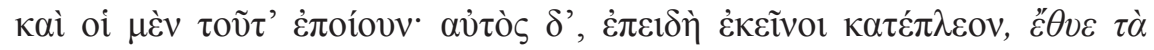

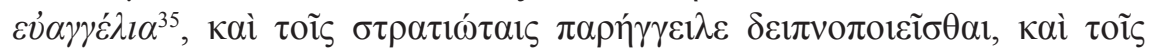



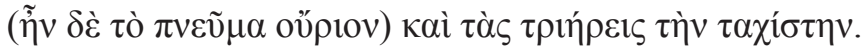

A Eteonico, una nave rápida de servicio le anunció todo lo sucedido en torno a la batalla naval. Pero él la despachó de nuevo ordenando a los que estaban dentro zarpar en silencio y no hablarlo con nadie y, de inmediato, navegar hacia su propio campamento coronados y gritando que Calicrátidas había vencido combatiendo en el mar y que todas las naves de los atenienses habían sido destruidas. Ellos hicieron esto y él, cuando aquellos se hicieron a la mar, celebró con sacrificios las buenas noticias, ordenó a los soldados cenar y a los comerciantes que, tras guardar sus mercancías

32 Sobre los sacrificios en campañas militares, Hutchinson (2000, p.47).

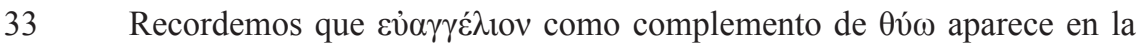
comedia.

34 Adviértase que la alusión a las buenas noticias ( $\varepsilon \dot{\alpha} \alpha \gamma \varepsilon \dot{\lambda} \lambda \alpha)$ está expresada por el verbo $\dot{\varepsilon} \xi \alpha \gamma \gamma \hat{\varepsilon} \lambda \lambda \omega$. П $\alpha \rho \alpha \gamma \gamma \hat{\varepsilon} \lambda \lambda \omega$, en cambio, alude claramente a mandatos.

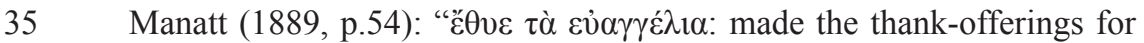
good news". 
en silencio en los barcos, navegaran a Quíos (pues el viento era favorable) y a las trirremes lo más rápidamente posible.

El objetivo de Eteonico era, en efecto, que sus hombres no se desalentaran ante el desastre acaecido y, para lograrlo, altera la información que recibe.

Con la misma intención manipula Agesilao las noticias de la derrota de los lacedemonios en la batalla de Cnido. En esta ocasión, cuenta Jenofonte que la flota comandada por Pisandro fue vencida por la dirigida por

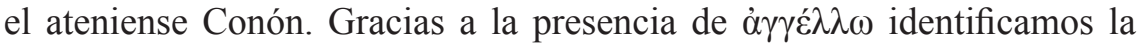
verdadera noticia:

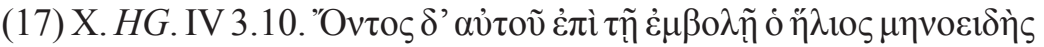

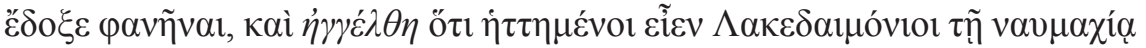

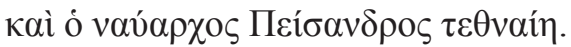

Cuando [Agesilao] estaba cerca de la entrada, el sol pareció mostrarse en forma de media luna. Y se anunció que los lacedemonios habían sido derrotados en la batalla naval y que el navarco Pisandro había muerto.

Estas son las nuevas que Agesilao recibe - junto al relato de cómo se desarrolló la batalla. Sin embargo, él mismo decide tergiversarlas:

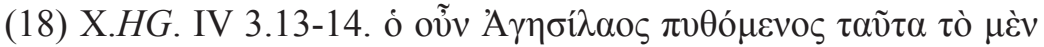

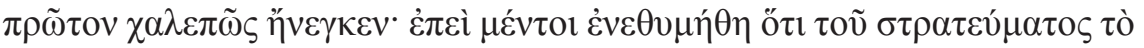

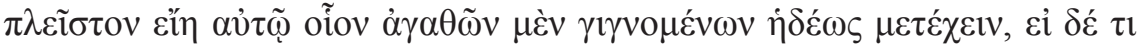



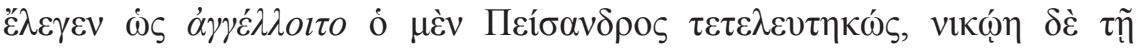

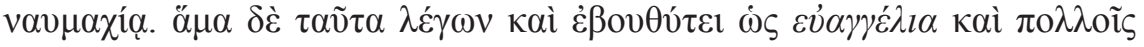

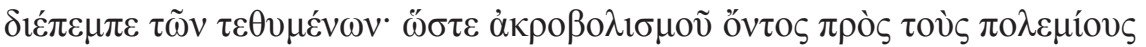

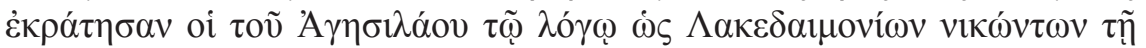

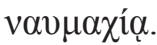

Agesilao, cuando se enteró de estas cosas, lo soportó verdaderamente mal. Sin embargo, después meditó que la mayor parte de su ejército era capaz de tomar parte con gusto en cuantas acciones resultan favorables, pero si veían alguna dificultad no tenían necesidad de participar y, dándole la vuelta a partir de esa reflexión, dijo que se le anunció que Periandro estaba muerto, pero había vencido en la batalla naval. Y al tiempo que decía estas cosas, además sacrificaba bueyes por las buenas noticias y distribuía entre muchos las víctimas de los sacrificios. Así, cuando se produjo una escaramuza contra los enemigos, dominaron los de Agesilao gracias a sus 
palabras, en la idea de que los lacedemonios habían vencido en la batalla naval.

Jenofonte atribuye a Agesilao $\alpha \gamma \gamma \varepsilon ́ \lambda \lambda \omega$ para comunicar a sus hombres la victoria ficticia que persigue que el ánimo de las tropas no decaiga.

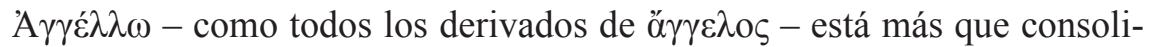
dado como término técnico para la transmisión de noticias y su presencia reviste de credibilidad las palabras de quien lo emplea. Por si fuera poco, Agesilao acompaña su embuste con sacrificios, lo que confiere a la escena mayor solemnidad ${ }^{36}$.

Como es lógico, la mayoría de noticias en las obras de los historiadores tienen que ver con la guerra. El léxico y el contexto nos ayudan a percibir los distintos matices. Por ejemplo, en (19) el complemento de $\dot{\alpha} \gamma \gamma \varepsilon \dot{\lambda} \lambda \omega \omega$ tiene tintes positivos:

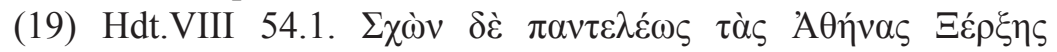

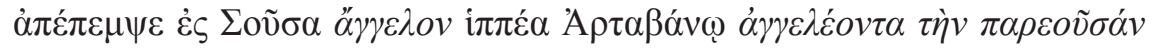
$\sigma \varphi \imath \varepsilon \dot{v} \pi \rho \eta \xi \xi i \eta v$.

Cuando se hubo apoderado por completo de Atenas, Jerjes envió un mensajero a caballo a Susa para que le anunciara a Artábano su actual éxito.

En cambio, en (20) ocurre todo lo contrario:

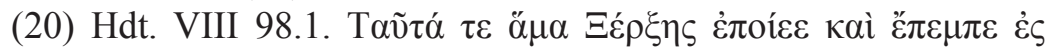

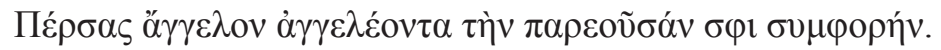

Al mismo tiempo que tomaba estas medidas, también envió a los persas a un mensajero para que les anunciara la desgracia acontecida.

La adversidad comunicada por este mensajero es la derrota de los persas ante los griegos en la batalla de Salamina.

En otros casos, la propia nueva es calificada como 'terrible', como

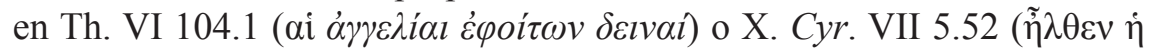
$\delta \varepsilon 1 v \grave{\alpha} \gamma \gamma \gamma \varepsilon \lambda i ́ \alpha)$.

$36 \quad$ Sobre este pasaje, Manatt (1889, p.202). 


\section{La oratoria}

Al estudiar los discursos de los oradores encontramos un pasaje de Sobre la corona de Demóstenes en el que él mismo pide que se lean unos versos de una tragedia, entre los que figura uno que contiene el verbo $\kappa \alpha \kappa \alpha \gamma \gamma \varepsilon \lambda \varepsilon \dot{\varepsilon} \omega$ :

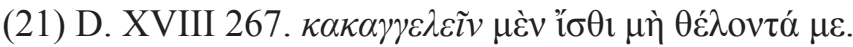

Que sepas que yo no quiero anunciar malas noticias.

No sabemos de qué tragedia podría haberse extraído este verso. Además, el pasaje ha sido muy discutido y, siguiendo a Goodwin, entre las

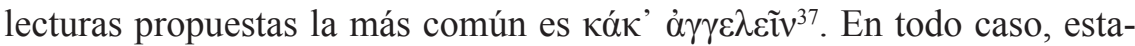
mos de acuerdo con López Eire ${ }^{38}$ en que el contenido del verso no resulta extraño a la tragedia en general, sino que responde a las expectativas del personaje literario del mensajero como portador de malas noticias.

En lo que al anuncio de buenas nuevas se refiere, en los textos de

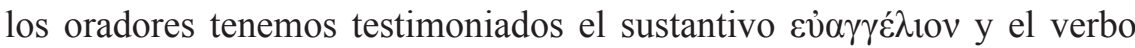


de Esquines ${ }^{39}$ y en el exordio del Areopagítico de Isócrates. Así reprocha el orador a su auditorio el hecho de que parezca haberse olvidado de los problemas de la ciudad:

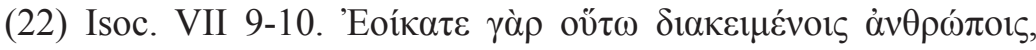

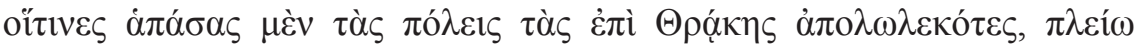

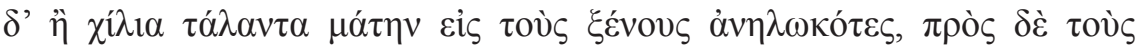



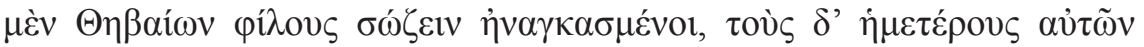

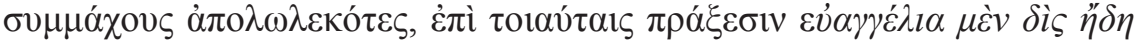

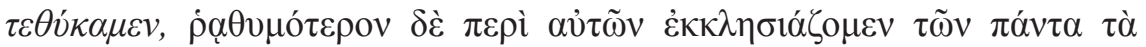



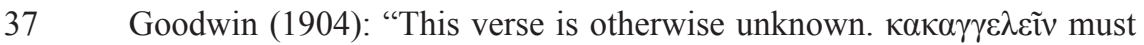

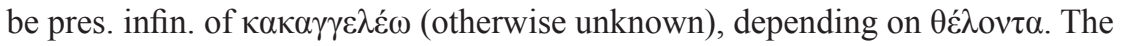

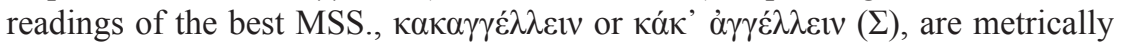
impossible. The common reading is кók' à $\gamma \gamma \varepsilon \lambda \varepsilon \tilde{v}$, an irregular fut. infin. with $\theta \varepsilon ́ \lambda o v \tau \alpha "$.

38 Véanse Abbott \& P. E. Mathenson (1899, p.104) o López Eire (1980).

39 Aeschin. III 160 (las nuevas también se celebran con sacrificios:




Pues sois semejantes a los hombres que se encuentran así y tras haber perdido todas las ciudades de Tracia, de haber pagado más de mil talentos de oro en vano a los extranjeros, de estar desacreditados entre los griegos, de haberos hecho enemigos del bárbaro e incluso de veros forzados a salvar a los amigos de los tebanos y de haber perdido a vuestros aliados por tales acciones ya hemos celebrado con sacrificios dos veces las buenas noticias y deliberamos en asambleas sobre ellas con más facilidad que quienes hacen todo lo que deben.

El pasaje está repleto de ironía y, una vez más, observamos cómo

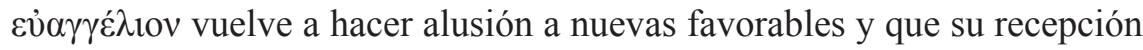
es celebrada con sacrificios, según refleja nuevamente el verbo $\theta v ́ \omega$.

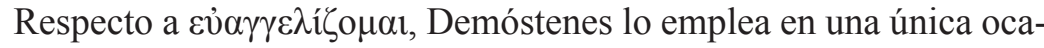
sión:

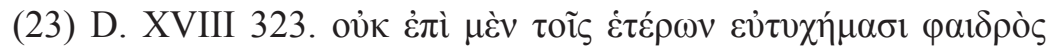

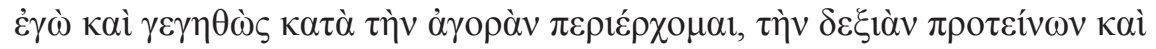



Yo no doy vueltas recorriendo el ágora alegre por éxitos de otros y regocijándome, tendiendo la mano a derecha y anunciando buenas noticias a los que creo que las van a anunciar allí (...).

Esta afirmación de Demóstenes se encuentra en el alegato que el propio orador realiza sobre su amor hacia Atenas y sus conciudadanos. Los otros a los que se refiere con $\dot{\varepsilon} \tau \dot{\varepsilon} \rho \omega v$ son los macedonios y Goodwin, en su comentario sobre este discurso demosténico, ha interpretado que el orador se está dirigiendo entre líneas a quienes pudieran informar en Macedonia de que el propio Demóstenes se alegraba por su victoria ${ }^{40}$.

En la oratoria, como en los textos de los historiadores, el concepto de noticia está estrechamente vinculado a lo público y la mayoría de nuevas tienen que ver con la guerra. En la $\pi$ ó $\lambda \iota \varsigma$, de igual modo que en el campo de batalla, es noticia la propia disensión y todo lo que de ella deriva: la muerte, los movimientos del enemigo, las victorias y las derrotas, los



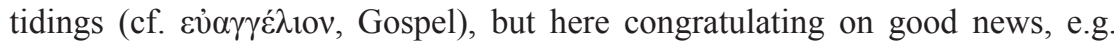
saying 'This is a great victory'. He means any of those who I ever think are likely to report thither (to Macedonia) such an event as my congratulating them on a victory of Alexander". 
tratados de alianza y de paz o las treguas. Todo ello es anunciado con los derivados de ö $\gamma \gamma \varepsilon \lambda$ o $\mathrm{y}$, de nuevo, tendremos que servirnos del contexto para dilucidar si las nuevas anunciadas son buenas o malas. Por ejemplo, en su Discurso Fúnebre ${ }^{41}$. Lisias afirma que las Amazonas no pudieron aprender de sus errores para, tras regresar a casa, anunciar ( $\dot{\alpha} \pi \alpha \gamma \gamma \varepsilon \tilde{\imath} \lambda \alpha \imath) \mathrm{su}$

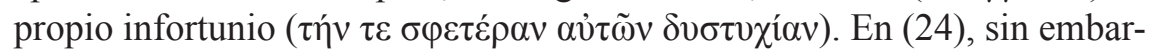
go, la noticia es favorable:



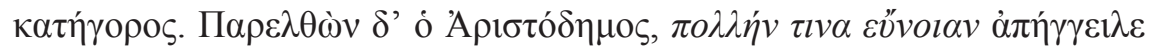

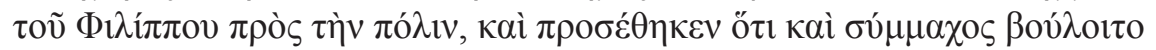

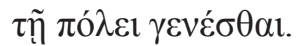

Y uno de los miembros del Consejo era Demóstenes, mi acusador. Y cuando compareció Aristodemo, anunció la buena disposición de Filipo hacia la ciudad y añadió que incluso quería ser aliado de la ciudad.

\section{Conclusiones}

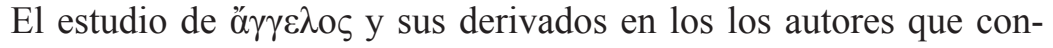
forman nuestro corpus evidencia que, aunque la gran mayoría de noticias transmitidas en ellos son adversas, no existe un derivado de ó $\gamma \gamma \varepsilon \lambda \circ \varsigma$ que designe la mala noticia. Sin embargo, sí hay uno para denominar la buena:

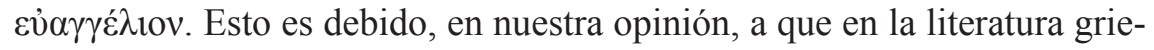
ga el concepto de noticia es un concepto de noticia mala (pensemos, por ejemplo, en la guerra y sus consecuencias en la Ilíada o los textos de los historiadores, la muerte - en la tragedia, sobre todo, los suicidios -, etc.) $\mathrm{y}$, por tanto, no es necesario crear una denominación específica, pues se da por supuesto que así es.

El hecho de que la nueva sea buena es, en cambio, algo novedoso, de ahí que para ella sí se haya generado un término concreto que solo aparece cuando las noticias son favorables. Esto es lo que sucede en la Odisea,

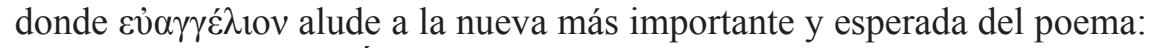
el regreso de Ulises a Ítaca. Por su parte, en la tragedia se presupone que el ó $\gamma \gamma \varepsilon \lambda$ o - figura creada expresamente para comunicar noticias que no podían representarse en escena - es portador de nuevas funestas $\mathrm{y}$, cuando



En la comedia aristofánica, las buenas - y absurdas - noticias tam-

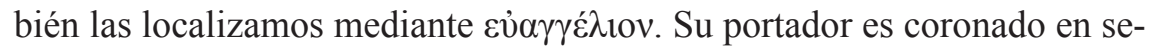

Cf. Lys.II 6. 
ñal de recompensa y las nuevas se celebran con sacrificios. Así, con un hecho que impregna sus palabras de seriedad y respeto, el transmisor de noticias consigue el efecto contrario, pues se trata de una excusa para parodiar al ő $\gamma \gamma \varepsilon \lambda o \zeta$ trágico, la solemnidad de sus anuncios y la escena que este protagoniza.

Los sacrificios para festejar la recepción de noticias favorables también son un recurso importante en los pasajes de Jenofonte y en los discur-

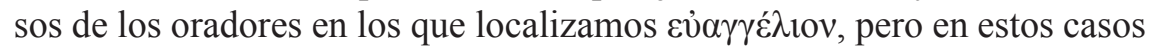
el propósito no es la burla. En las obras del historiador, el propósito es manipular nuevas relativas a la derrota para que los soldados no caigan en el desánimo. En la oratoria, el término aparece empleado como un recurso retórico con el que el orador impregna sus palabras de ironía.

Asimismo, aunque poco testimoniado (una vez en Aristófanes y otra en la oratoria) destaca en el corpus estudiado la presencia de $\varepsilon v \jmath \alpha \gamma \varepsilon \lambda i \zeta o \mu \alpha 1$ frente a la ausencia de un verbo que aluda expresamente a las nuevas desfavorables. En este sentido, contamos solo con un ejemplo localizado en su discurso Sobre la Corona en el que Demóstenes solicita la lectura de un

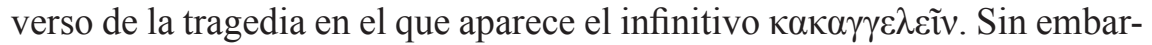
go, el pasaje es problemático $\mathrm{y}$, según parece, la lectura más aceptada no

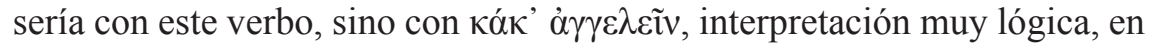
nuestra opinión, si atendemos a los datos que ofrece el examen del léxico

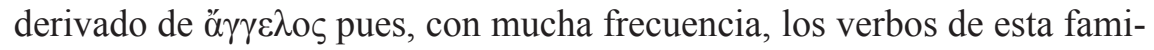
lia léxica nos permiten identificar las malas noticias presentando el neutro

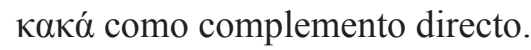




\section{REFERENCIAS BIBLIOGRÁFICAS}

ABBOtT, E. \& MATHESON, P. E. (1899). Demosthenes: On the Crown. Oxford: Clarendon Press.

BAILLY, A. (1973). Abrége du dictionnaire grec-français. Paris: Hachette.

BOWIE, A. M. (2014). Homer. 'Odyssey' Books XIII and XIV. Cambridge: Cambridge University Press.

BRIOSO SÁNCHEZ, M. (2006). "Algunas observaciones sobre el mensajero en el teatro ático clásico”. En E. C. Dorda et al. (eds.), Koinós lógos. (pp. 111-119). Murcia: Ediciones de la Universidad de Murcia.

COSERIU, E. (1982 [1962]). Teoría del lenguaje y lingüística general. Cinco estudios ( $3^{\mathrm{a}}$ ed. rev.). Madrid: Gredos.

DI GREGORIO, L. (1967). La scene d'annuncio nella tragedia greca. Milán: Vita e Pensiero.

DICKSON, J. P. (2005). "Gospel as News: $\varepsilon \dot{\alpha} \alpha \gamma \varepsilon \lambda-$ from Aristophanes to the Apostle Paul”, New Testament Studies, 51, 212-230.

EBELING, H. (1885). Lexicon Homericum. Leipzig: Teubner.

FORNIELES SÁNCHEZ, R. (2015). La transmisión de noticias en la literatura griega antigua [Tesis doctoral].

--- (2018). Sobre el mensajero trágico: propuesta de clasificación. Euphrosyne, XLVI, 27-44.

GARCÍA SANTOS, A. (2011). Diccionario del griego bíblico: Setenta y Nuevo Testamento. Estella: Verbo Divino.

GOODWIN, W. W. (1904). Demosthenes:De Corona. Cambridge.

HALLERAN, M. (2005). “Episodes”. En J. Gregory (ed.), A Companion to Greek Tragedy (pp. 167-182). Malden: Blackwell Publishers.

HARRISON, J. (2006). News. London: Routledge.

HUTCHINSON, G. (1985). Aeschylus: Septem contra Thebas. Oxford: Clarendon.

--- (2000). Xenophon and the Art of Command. London: Stackpole.

JONG, I. DE (2001). A Narratological Commentary on the Odyssey. Cambridge: Cambridge University Press.

LEWIS, S. (1992). "Public Information: News and Writing in Ancient Greece", Hermathena 152, 5-20.

--- (1996). News and Society in the Greek Polis. Chapel Hill: University of North Carolina Press.

LFGRE=SNELL, B. (1979-2010). Lexikon des frühgriechischen Epos. Göttingen: Vandenhoeck \& Ruprecht.

$L S J=H$. G. LIDDELL \& R. SCOTT (1968), A Greek - English Lexicon, 9th ed. Revised by H. S. Jones, Oxford.

LONGO, O. (1978). "Tecniche della comunicazione e ideologie sociali nella Grecia antica", Quaderni Urbinati Di Cultura Classica, 27, 63-92. 
LÓPEZ EIRE, A. (1980). Demóstenes. Discursos politicos I. Madrid: Gredos.

MANATT, I. J. (1889). Xenophon: Hellenica. Books I-IV. Boston: Ginn \& Company.

MASTRONARDE, D. J. (1979). Contact and Discontinuity: Some Conventions of Speech and Action on the Greek Tragic Stage. Berkeley: University of California Press.

--- (2002). Euripides Medea. Cambridge: University Press.

MELERO BELLIDO, A. (2000). “Comedia”. En J. A. López Férez (ed.), Historia de la literatura griega (pp. 431-499). Madrid: Cátedra.

MERRY, W. W., RIDELL, J. \& MONRO, D. B. (1886-1901). Commentary on Odyssey. Oxford: Clarendon.

MERRY, W. W. (1907). Homer. Odyssey, Books XIII-XXIV. Oxford: Clarendon. MILLS, S. P. (1981). "The Death of Ajax”, The Classical Journal, 76, 129-135. MITCHELL, A. M. (1836). The Knights of Aristophanes. London: Murray.

NEIL, R. A. (1901). The Knights of Aristophanes. Cambridge: University Press. PEREA MORALES, B. (1993). Esquilo. Tragedias. Madrid: Gredos.

RAU, P. (1967). Paratragodia. München: Beck.

SCHUDSON, M. (1978). Discovering News. New York: Basic Books.

SHOEMAKER, P. (2006). News \& Newsworthiness. Communications, 31, 10511.

SOMMERSTEIN, A. H. (1981). The Comedies of Aristophanes: Knights. Warminster: Aris \& Phillips.

STEPHENS, M. (1988). A History of News. Nueva York: Viking.

STROHM, H. (1959). "Beobachtungen zum Rhesus", Hermes, 87, 257-274. 